\title{
Des angles et des contours dans História do Cerco de Lisboa (une lecture parmi tant d'autres)
}

\author{
Armando AGUILAR DE LEÓN \\ Universidad Nacional Autónoma de México
}

"Esta é Lisboa prezada, Resguardada, Aqui terá perdição, O cristão".

José Saramago

Considéré un des meilleurs romanciers contemporains, José Saramago s'est fortement intéressé à la reconstitution fictionnelle de l'histoire: Levantado do chão (1980), Memorial do convento (1982), O ano da morte de Ricardo Reis (1984), A jangada de pedra (1986)... n'en sont que quelques exemples. Dans História do Cerco de Lisboa (1989), le futur prix Nobel (1998) récupère des épisodes historiques qui remontent à la fondation de son pays natal, le Portugal. Les événements 'fictionnalisés' se situent au Moyen Âge, à l'occasion des trois combats entre Maures et Chrétiens: la bataille d'Ourique (1139), la victoire de Santarém (1146) et la prise de Lisbonne (1147). Ces collisions se trouvent dans le cadre de $A$ Reconquista, période dans laquelle la chrétienté ibérique se bat contre les royaumes musulmans pour la récupération du territoire.

Cette matière première, qui appartient à l'historiographie officielle, subit chez Saramago un traitement métafictionnel. Une perspective extérieure du roman permet d'apprécier un univers diégétique angulaire où les événements de référence se multiplient sur plusieurs plans narratifs. Par ailleurs, le discours métafictionnel, loin de présenter un récit plat, contient des saillies autoréférentielles qui font ressortir à la surface du roman les procédés narratifs. Par contre, si l'on se place à l'intérieur de l'univers diégétique, on aperçoit que les événements historiques sont contenus dans plusieurs sphères se ressemblant entre elles, ayant tout de même des différences remarquables. Dans cette double perspective, le rôle du narrateur et celui du personnage principal se détachent.

En tenant compte de ce qui précède, notre analyse est organisée en deux parties. La première, à tendance descriptive, présente les caractéristiques postmodernes du roman ${ }^{1}$ sous la perspective de l'angularité. On y verra comment le discours métafictionnel

\footnotetext{
${ }^{1}$ Le roman postmoderne se caractérise par une 'fictionnalisation' d'épisodes historiques, fait qui lui a valu le terme de "métafiction historiographique" (Hutcheon, 1988). Cette catégorie narrative insiste sur le traitement de la matière historique et sur l'importance définitive d'un narrateur conscient
} 
met en relief une énonciation autoréférentielle et, par ailleurs, polyphonique. Ces caractéristiques donnent au récit une texture saillante. De plus, l'univers diégétique est composé d'angles résultant d'une superposition de niveaux narratifs qui plus tard, dans la partie deux, se révèleront comme des points d'intersection de sphères contenant chacune son propre univers diégétique et, en conséquence, ses propres coordonnées spatio-temporelles.

On proposera, dans la même deuxième partie, une hypothèse de lecture, disons, 'excentrique', par le fait qu'elle reste à la périphérie du centre de discussion et d'analyse que les critiques ont faites à História do Cerco de Lisboa. Cette hypothèse, si excentrique soit-elle, résulte de l'articulation d'éléments divers parsemés dans le texte: le cercle et le deleatur, le brouillard qui couvre Lisbonne, la co-habitation de personnages du passés et du présent dans la Lisbonne contemporaine et leur relation avec un acte transgressif du personnage principal. Celui-ci, correcteur d'édition, altère un texte historiographique et crée ainsi un monde uchronique ${ }^{2}$ où les événements officiels de la prise de Lisbonne se déroulent autrement. L'hypertexte de fiction bouscule ainsi l'hypotexte d'histoire. Si bien le bouleversement provoqué par le correcteur est très souvent abordé par la critique, une analyse de História do cerco de Lisboa sous la perspective de l' "uchronie' n'a pas encore été présentée, que l'on sache. Sans prétention d'originalité, je pars de la dite uchronie et mets en rapport direct le cercle et le deleatur comme symboles intradiégétiques de deux mondes parallèles. J'étends en outre ma réflexion jusqu'aux frontières de l'histoire frôlant la science-fiction. Je ne cherche pas à épuiser le sujet, je ne fais que présenter le résultat préliminaire d'une lecture que, comme les mondes parallèles, n'en est qu'une parmi tant d'autres.

\section{Des angles: l'univers diégétique et le discours métafictionnel}

História do Cerco de Lisboa est un roman de facture postmoderne qui recrée des événements historiques dans un univers complexe où la matière première prend une

de son rôle dans le roman, ce qui permet à l'auteur de construire un discours métafictionnel. De plus, les romanciers postmodernes intègrent une multiplicité de procédés narratifs — parmi lesquels la mise en abyme, la métalepse, l'autoréférentialité et la polyphonie- qui révèle la complexité d'un univers métadiégétique (Chanady, 1998).

${ }^{2}$ Le mot 'uchronie', calqué sur le mot 'utopie', fait référence à un sous-genre de la science-fiction qui propose un monde alterne; celui-ci se dégage d'un monde donné à partir d'un point de divergence; Fondanèche affirme: "Pour qu'il y ait Uchronie, l'auteur doit impérativement créer un incident sur l'axe historique, introduire une hypothèse plausible qui fait dériver le cours de l'Histoire" (2006: en ligne). Même si l'uchronie est chère aux romanciers de science-fiction dont leurs œuvres construisent un parcours historique spéculatif ou explorent la possibilité d'univers parallèles (théorie proposée en 1957 par le physicien américain Hugh Everett), la première manifestation datée du genre se trouve dans $A b$ Urbe condita libri (Ier siècle avant J.-C). Dans cette Histoire de Rome depuis sa fondation, Tite-Live imagine le territoire romain conquis par Alexandre le Grand. Au XVIIe siécle, Blaise Pascal invite à une réflexion uchronique: "Le nez de Cléopâtre, s'il eût été plus court, toute la face de la terre aurait changé" (Pensées). 
perspective multidimensionnelle. Cette perspective révèle une exposition angulaire des événements empruntés à l'histoire. Les angles superposés, l'univers diégétique devient inextricable. Le narrateur guide le lecteur à travers cette disposition irrégulière et lui en montre plusieurs détails. Ceci permet à ce dernier une meilleure appréciation de la profondeur de l'univers diégétique. Le narrateur y joue un rôle capital: il ne se limite pas à raconter l'histoire; il transgresse les frontières de l'énonciation et sa voix se fait l'écho d'une pluralité d'énonciateurs qui se trouvent placés sur les différents étages de la construction diégétique. D'ailleurs, la conscience de sa fonction dans cet univers particulier lui permet de faire de constants commentaires qui donnent au roman un caractère autoréférentiel et, en conséquent, métafictionnel, des traits principaux des romans postmodernes.

D'emblée, l'univers diégétique de História do Cerco de Lisboa contient un narrateur qui suit les mouvements de Raimundo Silva, personnage placé à Lisbonne au XXe siècle. Ce personnage, correcteur d'édition, en exercice de son métier, lit et corrige un texte historiographique intitulé 'História do Cerco de Lisboa'. La correction de ce texte l'engagera dans l'écriture d'un texte de fiction intitulé aussi 'História do Cerco de Lisboa'. On a donc affaire à une 'construction abymée', où les événements historiques de référence se projettent sur les différents plans narratifs. Apparemment, la mise en abyme comprend trois espaces ( $c f$. Schmidt, 2003: 55) où se déroule l'histoire du siège de Lisbonne: celui de Raimundo Silva, à Lisbonne, au XXe siècle; celui du texte de l'historien et celui de la postérieure écriture de Silva, les deux derniers se déroulant dans la Lisbonne médiévale. Néanmoins, entre le texte déclencheur (le texte historiographique) et la réécriture de Raimundo Silva, il existe un texte transitoire qui reste chez le personnage. Cette quatrième histoire du siège ${ }^{3}$ rend compte du processus de lecture du correcteur qui recrée, à sa manière, l'information présentée par l'historien:

Quando só uma visão mil vezes mais aguda do que a pode dar a natureza seria capaz de distinguir no oriente do céu a diferença inicial que separa a noite da madrugada, o almuadem acordou. [...] Não o tem descrito assim o historiador no seu livro. Apenas que o muezim subiu ao minarete e dali convocou aos fiéis à oração na mesquita, sem rigores de ocasião, [...] o miúdo pormenor não interessaria à histoira... Importaria saber, isso sim, é quem escreveu o relato daquele formoso acordar de almuadem na madrugada de Lisboa, com tal abundância de por menores realistas que chega a parecer obra de testemunha aqui presente... A

\footnotetext{
${ }^{3}$ Les dimensions de l'univers diégétique contiennent aussi deux récits complémentaires: l'histoire d'amour entre le correcteur et Maria Sara, son chef d'édition (plan premier); histoire qui a une projection sur les rapports entre Mogueime et Ouroana, personnages que Silva inclut dans sa version du siège (tiers plan; le deuxième récit est celui du texte historiographique et le quatrième, le plus profond, le texte transitoire qui se déroule chez Raimundo Silva).
} 
resposta, surpreendente, é que ninguém escreveu, que embora pareça que sim, não está escrito, tudo aquilo não foi mas que pensamentos vagos da cabeça do revisor enquanto ia lendo e emendando... (Saramago, 1989: 19; 22).

L'extrait commence par un récit minutieux du réveil de l' "almuadem" qui pressent l'aube juste avant le premier rayon de soleil. Le personnage aveugle traverse un long couloir et monte des escaliers en colimaçon; finalement il arrive en haut de la mosquée. En bas, il y a le repos de la ville et le fleuve reflétant le ciel: "a pele da água torna-se espelho do céu" (idem). Sans pouvoir apprécier la beauté matinale de Lisbonne, l'aveu$\mathrm{gle}^{4}$ convoque la ville à la prière. Le narrateur précise que ni le récit ni la description ne se trouvent dans le texte historiographique; l'historiographie ne s'intéressant point à ce type de détails. Il pose alors une question: 'qui a donc écrit ce récit à détails réalistes?' Réponse: personne, car ces minuties ne sont que de vagues pensées du correcteur qui révise le texte historiographique. Ceci révèle deux facultés essentielles du narrateur qui, placé sur un niveau métafictionnel, pénètre dans les recoins de chaque plan narratif et focalise internement le personnage, tout en faisant des signes au lecteur pour le faire remarquer les différences entre les plans narratifs.

Derrière ces facultés s'insinue un autre procédé définitif: la métalepse, qui implique, grosso modo, les glissements d'un niveau narratif vers un autre. Cette métalepse 'intérieure ${ }^{5}$ permet de comparer les faits présentés sur un plan (dans le cas ci-dessus, le texte historiographique) et les transformations de ces mêmes faits sur un plan alterne (la lecture du correcteur). Étant donné la superposition des plans narratifs, les métalepses intérieures sont un procédé constant qui permet les déplacements entre les différentes histoires du siège emboîtées.

Plus tard, le narrateur insiste sur le fait: ce qui se passe chez Raimundo Silva n'est pas écrit; "se escrita houvesse, e felizmente não há" (24). Il prend comme point de référence l'intertexte chez Silva (le texte transitoire) en rapport avec l'hypotexte historiographique. Cependant, à cause d'un jeu métafictionnel, le narrateur remonte sur un autre plan et pointe vers le roman dans lequel, lui même, il est le narrateur. Il finit par accepter: "não foi erro escrever, porque, enfim, escrito está, que era cego o almuadem" (29). Ceci dévoile l'autoréférentialité du roman qui explicite autant des aspects de l'univers diégétique que des procédés fictionnels utilisés par l'auteur.

\footnotetext{
${ }^{4}$ Le narrateur insiste que le "muezzin» est un personnage aveugle. Au fait, des romans de Saramago sont hantés par des personnages aveugles, ou qui subissent des périodes d'aveuglement, possédant la faculté de clairvoyance. C'est le cas de Blimunda dans Memorial do Convento. Le sujet de l'aveuglement prend place centrale dans Ensaio sobre a cegueira (1995), où, progressivement, les membres de toute une population deviennent, l'un après l'autre, tout d'un coup, aveugles.

${ }^{5}$ Dorrit Cohn, dans son brillant article "Métalepse et mise en abyme", détaille les différentes manifestations de la métalepse; en voici une précision importante: "Je nomme métalepse intérieure celle qui se produit entre deux niveaux de l'histoire elle-même, c'est-à-dire entre une histoire primaire et une histoire secondaire ou entre une secondaire et une tertiaire" (2003: en ligne).
} 
Il est évident qu'au sommet de cette mise en abyme angulaire, il est un espace qui privilégie les interventions du narrateur. Conscient de son rôle dans l'univers diégétique, il fait de longues digressions métafictionnelles. La causalité narrative suspendue par la profusion des commentaires, le récit prend des coins labyrinthiques. Le fil d'Ariadne est justement la voix du narrateur qui guide le lecteur dans cet univers dédaléen. ${ }^{6}$ Des expressions telles que "que ficasse o leitor sabendo" (19), "Era já tempo de sabermos quem" (31), "se nos lembrarmos de que" (157), "Tão largo rodeio da matéria não teve outra justificação que mostrar como" (278), parsemées tout au long du roman, sont un appel direct au lecteur; elles le préviennent de nouveaux angles dans la sinuosité du récit. Analysons l'une de ces expressions en contexte pour en tirer d'autres procédés impliqués:

Certos autores, quiçá por adquirida convicção ou compleição espiritual naturalmente pouco afeiçoada a indagações pacientes, aborrecem a evidência de não ser sempre linear e explícita a relaçâo entre o que chamamos causa e o que, por vir depois, chamamos efeito. Alegam esses [...] que... Indo mas longe... Enfim...

Esta conclusâo, que tem tanto de suspensiva como de providencial, permitenos, por hábil mudança do plano narrativo, regressar ao revisor Raimundo Silva (119-120).

Dans l'extrait, le narrateur suggère la progression discontinue du récit. Le sujet de la digression porte sur le fait de que 'certains auteurs détestent la non linéarité entre les causes et les conséquences'. Remarquons que la voix narratrice s'exprime à la première personne du pluriel ("chamamos"). Ce 'nous' implique premièrement le narrateur et en arrière-plan s'insinue la plume de l'auteur. C'est, en effet, l'auteur qui glisse, à travers la voix du narrateur, ses idées sur la causalité; c'est lui qui fait une critique de 'ces certains auteurs' qui construisent un roman linéaire où les causes et les conséquences s'enchaînent dans une progression cohérente. Ainsi, Saramago se sert-il de la voix narratrice pour livrer ses propres conceptions de l'écriture fictionnelle et justifier dans un acte autoréférentiel le traitement de la causalité dans son História do Cerco de Lisboa.

En outre, après la longue et ponctuelle dissertation ('ils soutiennent que', 'd'ailleurs', 'enfin'), le lecteur est alerté du passage de la réflexion métafictionnelle vers le plan narratif premier, où Raimundo Silva agit. L'incise 'por hábil mudança do plano narrativo' révèle une métalepse extérieure ${ }^{7}$ qui déplace narrateur et lecteur du sommet métafictionnel à l'intérieur du récit fictionnel.

\footnotetext{
${ }^{6}$ Dans Todos os nomes (1997), le labyrinthe est un espace imaginaire projeté sur l'espace physique (l'arrière-chambre d'un bâtiment administratif et le cimetière). Dans ce roman, le personnage, José, s'engage dans la quête d'une femme décédée.

7 "Je nomme métalepse extérieure celle qui se produit entre le niveau extradiégétique et le niveau diégétique, c'est-à-dire entre l'univers du narrateur et celui de son histoire" (Cohn, 2003: en ligne).
} 
L'énonciation 'transvocalisée' est un procédé de premier ordre dans cette mise en abyme; elle ne se confine pas dans le niveau supérieur de l'univers diégétique, communiquant auteur, narrateur et lecteur. Au-dessous de la surface, la transvocalisation permet de véhiculer les voix qui se prononcent dès tréfonds de la mise en abyme. Il ne faut pas oublier que Raimundo Silva lit une 'História do Cerco de Lisboa' écrite par un historien; bien que celui-ci n'apparaisse dans le roman qu'en deux ou trois occasions, son texte est toujours là, comme point de repère pour Raimundo Silva. Même si dans l'extrait suivant le narrateur est toujours le détenteur de la parole, c'est à travers la lecture de Silva que la voix de l'historien émerge: "Nesta última página da História do Cerco de Lisboa pode Raimundo Silva encontrar a ardente expressão de um patriotismo fervoroso [...] repare-se no que escreveu o historiador. No alto do castelo o crescente muçulmano desceu pela derradeira vez e, definitivamente, para sempre, ao lado da cruz que anunciava ao mundo o baptismo santo da nova cidade cristã...” (40-41).

Dans cet univers où résonnent plusieurs voix, Raimundo Silva y fait sentir la sienne: lorsqu'il corrige le texte historiographique d'une manière critique, il analyse le discours de l'historien; lorsqu'il se laisse aller par les événements racontés, il fait des fabulations. En tout cas, Silva participe activement de cette polyphonie.

Ayant exposé les principaux traits postmodernes de la História do Cerco de Lisboa (mise en abyme, intertextualité, métalepse, discours métafictionnel et autoréférentiel et polyphonie), continuons avec la partie qui porte sur les contours pour observer l'importance du cercle et le deleatur dans la fiction uchronique.

\section{Des contours: le cercle et le deleatur ou les univers parallèles du siège de Lisbonne}

Comme l'on a déjà remarqué, dans la fiction de Saramago la matière historique se projette sur des différents plans narratifs superposés, dont l'ensemble donne une perspective pluridimensionnelle. Si l'on a l'impression de pénétrer dans un univers diégétique angulaire c'est à cause de cette superposition et du caractère métafictionnel du discours. Cependant, placé à l'intérieur du récit fictionnel, on aperçoit que les événements historiques de référence sont concentrés dans des micro-univers ayant chacun sa propre diégèse. La superposition angulaire des plans narratifs se révèle ainsi comme des points d'intersection entre des aspects spatio-temporels des différentes sphères.

L'un de ces micro-univers se trouve contenu dans le texte historiographique. Celui-ci raconte comment à l'occasion du siège de Lisbonne, les croisés aident le roi Afonso Henriques à la prise de la ville sous domination musulmane. Dans ce sujet qui fait partie de l'historiographie officielle, l'historien introduit sans le savoir une séquence ininterrompue d'anachronismes. Les éléments historiques mal placés finissent par énerver le correcteur, qui, dans un acte transgressif, altère à son tour le texte de l'historien 
par l'introduction d'une négation: 'les croisés n'aident pas le roi Afonso Henriques à la prise de la Lisbonne maure'. Cette transgression change le cours de l'histoire: le correcteur crée ainsi un univers parallèle, dont le bouleversement historique aura des répercussions sur l'univers que Raimundo Silva habite.

Mais l'acte transgressif et le parallélisme entre la 'História do Cerco de Lisboa' de l'historien et la version de Silva qui s'en dégage sont annoncés depuis le début du roman. Le premier chapitre de História do Cerco de Lisboa se détache de l'ensemble du roman par le fait d'être une scène où se déroule une «conversação preambular e algo labiríntica» (39) dans laquelle le narrateur n'intervient qu'au début pour introduire le dialogue: "Disse o revisor" (11). Ensuite, les seules voix qui s'écoutent sont celles du correcteur et de l'historien entretenant une conversation ( $c f .11-16)$. Le sujet porte sur un signe typographique qui marque un effacement, une suppression à effectuer dans un texte: "Sim, o nome deste sinal é deleatur, usamo-lo quando precisamos suprimir e apagar, a própria palavra o está a dizer, e tanto vale para letras soltas como para palavras completas" (idem). Le signe ' $\phi$ ', dont le nom d'origine latine, 'deleatur', signifie 'qu'il soit détruit', est comparé à un serpent qui se repent juste avant de se mordre la queue: "Lembra-me uma cobra que se tivesse arrependido no momento de morder a cauda" (idem). Il est évident que l'historien fait allusion à l'Ouroboros, symbole circulaire d'origine égyptienne qui représente le 'temps cyclique', 'l'éternité', 'la perfection', 'la continuité inlassable', 'le perpétuel retour' (vid. Chevalier, 1982: 716). Ainsi, le cercle et le deleatur sont-ils rapprochés et opposés: le premier comme l'équilibre dans la continuation d'une ligne qui se répète à jamais; le deuxième comme une ligne circulaire qui avant de compléter le circuit, change sa trajectoire. Le deleatur détruit ainsi la continuité et l'équilibre du cercle. Même si les personnages ne font pas de référence explicite à l'Ouroboros, son symbolisme se présente dans la conversation; mais les attributions positives du symbole ancien prennent chez le correcteur une tournure négative: "até uma serpente hesitaria diante da eternidade" (Saramago, 1989: 11); la perfection circulaire devient dans les mots du correcteur "un terrível círculo" (idem). De la comparaison/opposition entre le cercle et le deleatur, les personnages dérivent vers 'le plaisir de la transformation'; puis, ils passent sur le métier du correcteur: "sempre chega o dia em que é preciso corrigir mais no profundo [...] as piores dificuldades resolvo-as à maneira expedita, escrevendo uma palavra por cima de outra" (12). Après ces mots prophétiques, les personnages s'engagent dans une discussion sur le caractère de l'histoire: l'un soutient que l'histoire fut de la vie réelle; l'autre le contredit: l'histoire est littérature: "em minha discreta opinião, senhor doutor, tudo quanto não for vida, é literatura, A história também, A história sobretudo" (15). L'historien maintient sa position. Le correcteur conclut: "Que seria de nós se não existisse o deleatur" (16). Le deleatur encadre alors la conversation préliminaire, où s'opposent, en plus, deux conceptions de l'histoire. 
Le caractère négatif de la circularité se réitère plus tard. À l'occasion d'une incursion "pelas fronteiras da entomologia" (27), l'allégorie sur Aristote et la mouche renferme une critique contre la doctrine: la faute du maître sera reprise de manière dogmatique par les épigones, même si leur expérience dans la vie réelle contredit l'autorité:

[...] a afirmação do sábio Aristóteles de que a mosca doméstica comum tem quatro patas, redução aritmética que os autores seguintes vieram repetindo por séculos e séculos, quando já as crianças sabiam, por crueldade e experimentação, que são seis as patas da mosca, pois desde Aristóteles as vinham arrancando, voluptuosamente contando, uma, duas, três, quatro, cinco, seis, mas essas mesmas crianças, quando cresciam e iam ler o sábio grego, diziam uma para as outras, A mosca tem quatro patas, tanto pode a autoridade magistral, tanto sofre a verdade (idem).

Cette allégorie insiste sur la circularité vicieuse, 'le terrible cercle', dans les mots de Raimundo Silva. L'apport du narrateur s'applique au texte de l'historien dont les anachronismes ne sont qu'une continuation des erreurs de ses prédécesseurs. Étant donné la circularité de l'histoire, ces anachronismes seront repris par les successeurs: "Amanhã irão dizer os leitores inocentes e repetirá a juventude nas escolas que a mosca tem quatro patas [...], e no próximo centenário da tomada de Lisboa aos mouros, [...], não faltará um presidente para evocar aquela suprema hora em que...” (43). Ainsi, l'histoire dans sa circularité est-elle conformée de fautes dont l'origine et la fin se perdent au fil du temps.

La lecture de Silva constate cette répétition inlassable: "Em quatrocentas e trinta e sete páginas não se encontrou um facto novo, uma interpretação polémica, un documento inédito, sequer uma releitura. Apenas mais uma repetição das mil vezes contadas e exaustas histórias do cerco" (39). Silva lui-même se voit pris dans le cercle: "Está como fascinado, lê, relê, torna a ler a mesma linha, esta que de cada vez redondamente afirma que os cruzados auxiliarão os portugueses a tomar Lisboa" (48). Devant la rondeur de cette assertion qui ferme l'ouroboros de l'histoire officielle du Portugal, il se glisse chez Silva une idée qui le met hors de lui. Perturbé jusqu'aux extrêmes de la folie, le correcteur souffre un dédoublement. ${ }^{8}$ Il se livre alors une lutte entre “o campeão angélico e o campeão demoníaco" (49) entre 'Dr. Jekill et Mr. Hyde’: "Mas esta batalha [...] vai ganhá-la Mr. Hyde [...] com a mão firme (Silva) segura a estereográfica e acrescenta uma palavra que o historiador não escreveu, que em nome da verdade histórica não poderia ter escrito nunca, a palavra Não, agora o que o livro passou a dizer é que os cruzados Não auxiliarão aos portugueses a conquistar Lisboa, assim está escrito e portanto passou a ser verdade" (49-50).

\footnotetext{
${ }^{8}$ Ainsi que l'aveuglement, la duplicité du moi est un sujet cher à Saramago. Il explorera la problématique de l'identité et le dédoublement dans $O$ homem duplicado (2002).
} 
Ce 'Não' de Silva nuit à la circularité perpétuelle de l'histoire du siège; la ligne circulaire prend une autre trajectoire: 'Qu'il soit détruit', dit le deleatur et Silva bouleverse l'histoire officielle en appliquant à sa circularité un 'Não', un point de divergence: un univers parallèle est créé. Après avoir troublé l'histoire, Silva considère de rétablir l'ordre en effaçant le point de divergence: "o mundo regressará à antiga e tranquila órbita, o que foi continuará a ser" (51). Il est trop tard... Le monde uchronique est déjà en marche.

Les répercussions de ce bouleversement du monde médiéval officiel se font ressentir sur la Lisbonne du XXe siècle: une sorte de trou temporel instable, symbolisé par le dense brouillard, couvre par moments la ville. Il est vrai que le brouillard se trouve dans História do Cerco de Lisboa bien avant le point de divergence; mais il faut tenir compte de que la lecture de Silva avant l'introduction du célèbre 'Não' est l'antécédent de cet univers parallèle. On pourrait dire que celui-ci se trouve en gestation depuis que Silva a commencé la lecture du texte historiographique; l'introduction du 'Não' est sa confirmation. D’ailleurs, le phénomène météorologique devient le sujet de conversation après le bouleversement provoqué par Silva; dans les rues, les gens le commentent vivement (63). Enfin, pour renforcer notre argument, donnons le contexte du tout petit extrait qui suit: dans une crèmerie, une grosse femme gourmande se livre aux plaisirs de la pâtisserie portugaise, son biscuit s'émiette: "os fragmentos do mil-folhas [...] são assim como uma poeira cósmica, incontáveis, gotículas de um nevoeiro infinito" (idem). Les mies du biscuit sont comparées à la poussière cosmique, à un brouillard infini... Le brouillard qui cerne la ville de Lisbonne prend alors une dimension minimale et cosmique à la fois.

Par ailleurs, l'épisode de la crèmerie est remarquable puisqu'on y assiste à un des points d'intersection entre la Lisbonne assiégée et la Lisbonne contemporaine: Silva descend dans les rues et se dirige vers la crèmerie; le narrateur explique: "Evidentemente, a Leitaria A Graciosa, onde o revisor agora vai entrando, não se encontrava aqui no ano de mil cento e quarenta e sete em que estamos" (61). Le narrateur participe de la déstabilisation. On voit Raimundo Silva marcher vers la crèmerie, mais le narrateur dit qu'on est en 1147. Les références temporelles se heurtent les unes contre les autres. Lisbonne alors est le point d'intersection de deux univers. Ses rues sont peuplées des personnages sortis du passé musulman qui côtoient les habitants de la Lisbonne contemporaine. La tranquillité des Lisbonnais du XXe siècle contraste avec la violence du siège médiéval. Les personnages de 'A Graciosa' se trouvent à cheval entre deux époques, eux-mêmes sont des Portugais contemporains et des Maures médiévaux à la fois. Voici la description du narrateur, où se réunissent les voix des personnages: VA cidade está que é um coro de lamentaçôes, com toda essa gente que vem entrando fugida, enxotada pelas tropas de Ibn Arrinque, ${ }^{9}$ o Galego, que Alá o fulmine e o condene ao

\footnotetext{
9 'Ibn Arrinque', nom arabe de 'Afonso Henriques', 'le fils de Henrique'.
} 
inferno profundo, e vem em lastimoso estado os infelizes, escorrendo sangue de feridas, chorando e gritando [...] é o aviso que manda diante o rei português, E parece, disse o dono da leitaria, que vêm cruzados por mar, malditos sejam eles" (61).

L'horreur du siège et les personnages de la crèmerie n'est qu'un exemple des plusieurs points d'intersection résultant de la transgression créatrice du correcteur.

Raimundo Silva continue son rôle de démiurge en apportant l'équilibre à cet univers bouleversé. Il entreprend la réécriture de l'histoire du siège de Lisbonne. Le texte de l'historien à la main, comme point de repère, Silva parcourt les rues de la ville. Lisbonne contemporaine devient un document historique ouvert aux yeux du personnage: 'os registos de azulejos', les différents arcs, les légendes chrétiennes sur les miracles do Santo António, l'endroit occupé par les anciennes murailles ${ }^{10}$ de la ville médiévale... En évitant de répéter les mêmes erreurs que l'historien, Silva va chercher d'autres sources pour sa réécriture. Il réussit à faire de son histoire alterne un monde cohérent. L'équilibre entre les sphères est rétabli... Vers la fin du roman, le cosmos rentre dans son perpétuel apaisement, le chaos est banni. La réécriture de Silva redonne à l'univers son équilibre. ${ }^{11}$

\section{Conclusion}

Cette exploration des angles et des contours dans História do Cerco de Lisboa nous a permis de remarquer les caractéristiques postmodernes du roman et le caractère uchronique de l'histoire. En parlant des procédés narratifs, insistons sur la caractéristique la plus évidente de l'univers diégétique: la mise en abyme. Dans cet univers abyssal irrégulier, le narrateur occupe une place privilégiée. Situé au sommet de la construction, il domine les différents plans du roman. Aussi, peut-il digresser sur la surface du récit que pénétrer les endroits les plus profonds de la pensée du personnage. C'est la métalepse, procédé qui revient fréquemment, l'artifice qui lui permet de descendre et de surmonter les différents étages superposés de la mise en abyme. Par ailleurs, le narrateur est conscient des problèmes que ses longs commentaires et ses continuels déplacements peuvent poser au lecteur; il lui fait alors des constants appels pour le prévenir des changements imminents et le situer sur le plan correct. Ainsi, le narrateur se sert-il d'un discours métafictionnel qui révèle le caractère autoréférentiel du roman. Encore, à travers ces va-et-vient, la voix narratrice sert de support à une pluralité

\footnotetext{
${ }^{10}$ Dans la traduction du titre en français on perd la double référence du mot portugais 'cerco', qui envoie à la fois au siège des croisés et aux murailles qui entouraient la ville médiévale.

${ }^{11}$ Par ailleurs, Silva rencontre l'amour. Même si le rôle de Maria Sara dans le réécriture de Silva n'est pas à négliger, les rapports entre ces personnages appartiennent à un autre sujet.
} 
d'énonciateurs qui peuplent l'univers angulaire. Bref, mise en abyme, intertextualité, métalepse, autoréférentialité et polyphonie sont les traits caractéristiques du roman. Leur présence explicite représente de constantes saillies dans le discours narratif.

En ce qui concerne les contours, le cercle et le deleatur sont une sorte de symboles intradiégétiques qui représentent le texte historiographique et sa variante uchronique. Aussi, la 'História do Cerco de Lisboa' de l'historien et son homonyme en version fiction du correcteur devenu écrivain se côtoient-ils de tout près. De plus, l'intersection de ces circonférences met en relief des épisodes historiques se déroulant dans une temporalité décalée. La présence constante du brouillard est aussi intrigante, il symbolise un trou cosmique qui, se cernant sur la Lisbonne d'aujourd'hui, réunit des personnages médiévaux et contemporains. Raimundo Silva agit alors en démiurge en créant une causalité cohérente dans le monde qu'il a créé. De cette manière, le présent et l'histoire retrouvent leur stabilité.

Sous la perspective des mondes uchroniques, la conjonction des éléments analysés dans les deux chapitres pourrait envoyer à des épisodes chers à la science-fiction, sans en être História do Cerco de Lisboa une 'parodie filée'. La mise en abyme se révèlerait ainsi comme un grand cosmos, une métadiégèse ( $c f$. Chanady, 1998: en ligne) où orbitent des mondes divers qui, à cause de l'intertextualité, se rapprochent et s'écartent à la fois. Dans cette métadiégèse, la métalepse serait le procédé qui permet les points d'intersection des mondes parallèles, une sorte de 'trou de ver' dans lequel narrateur et lecteur glissent entre un monde et un autre. La polyphonie, à son tour, représente l'expression des habitants de divers mondes.

Saramago dans História do Cerco de Lisboa présente une réflexion sur l'histoire et problématise l'écriture historiographique. Sa critique vise le manque d'originalité des textes qui ne font que répéter les mêmes épisodes écrits depuis le Moyen Âge. Saramago revisite l'épisode de la fondation du Portugal et lui donne une nouvelle impulsion en fictionnalisant ses passages. Aussi, les personnages sont-ils sortis du contexte original et replacés sur la Lisbonne d'aujourd'hui. L'écrivain portugais fait, de cette manière, revivre l'histoire. Enfin, Saramago déploie les beautés de Lisbonne, ville qui devient un document authentique pour la réécriture de Raimundo Silva.

\section{Oeuvres citées}

CHANADY, Amaryll. 1998. "Une métacritique de la métalittérature”. Etudes françaises. Université de Montréal, Université Laval et Université de Québec à Montréal. En ligne: ${ }^{12}$ http://www.erudit.org/revue/etudfr/1987/v23/n3/035732ar.pdf

Chevalier, Jean et Alain GheErbrant. 1982. Dictionnaire des symboles. Paris: Robert Laffont / Jupiter.

\footnotetext{
${ }^{12}$ Tous les ‘e-docs' ont été consultés en février 2007.
} 
CoHn, Dorrit. 2003. "Métalepse et mise en abyme". Vox poética. Théorie et critique. Centre de recherches sur les arts et le langage. En ligne: http://www.vox-poetica. com/t/metalepse.htm

FONDANECHE, Daniel. 'L'Uchronie comme moteur de l'Histoire (im)possible: L'Appel du 17 juin d'André Costa". La science fiction dans l'histoire, l'histoire dans la science fiction. Cycnos, Centre de Recherches sur les Ecritures de Langue Anglaise de la Faculté des Lettres de l'Université de Nice Sophia Antipolis. En ligne: http:// revel.unice.fr/cycnos/document.html?id=603

HutCHEON, Linda. 1988. "Historiographic Metafiction". The Canadian Postmodern. A Study of Contemporary English-Canadien Fiction. Toronto: Oxford University Press.

Saramago, José. 1989. História do Cerco de Lisboa. Lisboa: Caminho.

SCHMIDT, Júlia Marina da Graça. "Manual de pintura e caligrafia, história do cerco de lisboa e o evangelho segundo jesus cristo -uma leitura trilológica". Romansk forum (2003-1). Universitetet i Oslo. En ligne: http://www.duo.uio.no/roman/Art/ Rf17-03-1/04.Schmidt.pdf 